\title{
Editoriale Milleproroghe e principi contabili internazionali: un errore "nazionale"
}

\author{
Alberto Quagli*
}

Fin dagli esordi dell'applicazione obbligatoria degli IFRS in Europa, vi furono alcuni studiosi che paventarono quanto la promessa standardizzazione contabile fosse una chimera, minacciata da divergenti incentivi nazionali, da diversi meccanismi di enforcement, da diverse tradizioni contabili (per tutti Ball, 2006; Nobes, 2006 ${ }^{1}$ ).

Recentemente si è registrata in Italia una modifica normativa che esplicita in modo palese quanto gli IFRS possono essere diversamente interpretati in funzione delle specificità nazionali.

Per chi si occupa di bilanci aziendali, colpisce infatti la notizia che nel Decreto di Conversione del "Milleproroghe" (DL 29 Dicembre 2010, n. 225 convertito in Legge n. 10 del 26 Febbraio 2011) sia stata approvata la norma di cui all'art. 2, comma 26 che va a modificare il testo del D.Lgs 38/2005 riguardante l'applicazione nel nostro Paese dei principi contabili internazionali. Con un colpo di spugna, quelle poche righe possono vanificare sei anni di sforzo progressivo verso la corretta implementazione dei principi contabili internazionali nei bilanci delle aziende.

In sostanza la citata norma consente al Ministero della Giustizia la potestà di emanare "disposizioni applicative" dei Regolamenti Europei entrati in vigore successivamente al 31 dicembre 2010 che adottano i principi contabili internazionali. Queste disposizioni applicative, emanate di concerto con gli organismi contabili nazionali (OIC, Banca d'Italia, Consob e ISVAP), avrebbero lo scopo di "realizzare ove compatibile, il coordinamento tra i principi medesimi e la disciplina di cui al titolo $\mathrm{V}$ del libro $\mathrm{V}$ del codice civile, con particolare riguardo alla funzione del bilancio di esercizio".

* Editor di Financial Reporting.

1. Ball R. (2006), International Financial Reporting Standards (IFRS): Pros and Cons for Investors. Accounting and Business Research, 36 (Special Issue), pp. 5-27; Nobes C.W. (2006), The survival of international differences under IFRS: towards a research agenda. Accounting and Business Research, 36, n. 3, pp. 233-245. 
Alberto Quagli

Le perplessità sollevate da tale norma sono rilevantissime, per vari ordini di ragioni.

Sotto il profilo formale, si deve anzitutto rilevare che i Regolamenti Europei sono immediatamente esecutivi nell'impianto normativo italiano, diversamente dalle Direttive che vanno recepite tramite atto parlamentare. Sottoporre tali Regolamenti a "disposizioni applicative", espressione che non chiarisce certo bene i contorni del possibile intervento nazionale, è una prima forzatura.

In secondo luogo, trattandosi di principi contabili internazionali dello IASB, gli addetti ai lavori sanno bene che l'unico organismo deputato a fornire interpretazioni applicative sui principi stessi è l'IFRIC (International Financial Reporting Interpretations Committee). Si può non essere d'accordo su tale circostanza, ma applicare in un bilancio una interpretazione proveniente da organo diverso dall'IFRIC priva il bilancio stesso della essenziale caratteristica di essere "conforme agli IFRS" per trasformarlo potenzialmente in un ibrido, con reali problemi di accettazione da parte degli organismi di borsa.

In terzo luogo sembra assurdo che il faro delle "disposizioni applicative" menzionate nel Milleproroghe sia rappresentato dalla "funzione del bilancio di esercizio" come stabilita nel Codice Civile. Ci si dimentica forse che tale funzione è quella che ha stabilito l'Unione Europea nel lontano 1978. Che è poi la stessa Istituzione che decide di recepire gli IFRS in quanto non contrastanti con il principio ultimo della rappresentazione "chiara, veritiera e corretta" della situazione aziendale (per dirla all'italiana). Per cui che senso ha sbandierare il primato della funzione del bilancio inclusa nel Codice Civile se poi la stessa funzione si deve presumere che sia stata già tutelata tramite il vaglio preventivo che la Commissione Europea adotta quando esamina gli IFRS prima di recepirli ? Forse perché non ci fidiamo dell'Europa ?

Sotto il profilo formale, quindi, la norma del Milleproroghe, per quanto proposta in toni sommessi ("disposizioni applicative") non regge, è debolissima, e può essere considerata anche contraria all'ordinamento dell'Unione Europea.

Sotto il profilo operativo, poi, si apre il campo ad una serie di problematiche tutte da definire. Ad esempio, la maggior parte dei Regolamenti Europei che adottano gli IAS, riguardano modifiche ai principi contabili già esistenti, non principi completamente nuovi. Questo significa che la citata norma estende agli organi legislativi nazionali il potere di intervenire sull'intero principio che viene modificato dal Regolamento o solo sulle disposizioni innovate? Oppure ancora: la norma riguarderà solo l'applicazione degli IFRS nel bilancio separato/Individuale o anche in quello consolidato? Il richiamo alla norma civilistica può prestarsi ad essere usato per entrambi contesti.

Al di là di queste considerazioni "tecniche", il problema, come in tutte le cose, sta nella sostanza. Quale ratio si cela dietro tale norma? Probabilmente l'idea che certi IFRS o certe specifiche disposizioni in essi contenute possano 
non piacere. Vuoi perché si ritiene ledano il principio di conservazione del capitale, vuoi perché costringono le imprese a complicate sofisticazioni per valutare elementi un tempo di "semplice" evidenza (valutazione dei debiti al valore nominale, ecc.).

Si può anche discutere per dieci anni di fila, e noi accademici siamo maestri in questo, su cosa ci sia di "giusto" per noi italiani negli IFRS e cosa di "sbagliato". Ma che l'impianto dei principi contabili internazionali (IAS/IFRS) fosse lontano mille miglia dalla nostra tradizione contabile, ispirata al principio della prudenza valutativa e della realizzazione finanziaria dei ricavi, era palese ben prima dal 2005, quando il Legislatore Italiano con il famoso D.Lgs. 38 decise di estendere l'applicazione degli IFRS anche ai bilanci separati e individuali, e non solo al bilancio consolidato come fatto dalla maggior parte dei Paesi Europei.

Sono i bilanci separati e individuali e non quelli consolidati ad essere usati dal fisco per tassare le imprese, e dalle assemblee, per decidere i dividendi. In quanto tali, essi sono i bilanci con impatto sostanziale a livello nazionale decisamente maggiore.

Perciò quello che sarebbe da ridiscutere è l'applicazione in Italia degli IAS/IFRS anche ai bilanci della singola società, in via obbligatoria per tutte le banche, le assicurazioni e le società quotate e in via facoltativa ad una pletora di altre aziende, come previsto dal D.Lgs. 38/2005. Quello è stato l'errore primigenio, gratuito in quanto non richiesto dall'Unione Europea che lasciava invece agli Stati membri una facoltà e non un obbligo di applicare gli IFRS anche nei bilanci separati.

Su tale errore mai la politica e le istituzioni pubbliche italiane hanno mostrato apertamente di riflettere in modo serio.

Adesso tamponare con fumose "disposizioni applicative" approvate con Decreto Ministeriale le "falle" o le "stranezze" degli IFRS rispetto alla logica contabile nazionale e agli interessi legali e istituzionali del nostro Paese, oltre che scorretto legalmente, sembra quasi una tardiva e malcelata rivendicazione di bottega. 